\title{
INTEGRATION ON ARTIN TORIC STACKS AND EULER CHARACTERISTICS
}

\author{
DAN EDIDIN AND YOGESH MORE \\ (Communicated by Lev Borisov)
}

\begin{abstract}
There is a well-developed intersection theory on smooth Artin stacks with quasi-affine diagonal. However, for Artin stacks whose diagonal is not quasi-finite, the notion of the degree of a Chow cycle is not defined. In this paper we propose a definition for the degree of a cycle on Artin toric stacks whose underlying toric varieties are complete. As an application we define the Euler characteristic of an Artin toric stack with complete good moduli space, extending the definition of the orbifold Euler characteristic. An explicit combinatorial formula is given for 3-dimensional Artin toric stacks.
\end{abstract}

\section{INTRODUCTION}

Let $\mathcal{X}$ be complete Deligne-Mumford stack. There is a well-developed intersection theory on such stacks [Vis, EG98, Kre which includes the notion of the degree of the 0 -cycle. Over an algebraically closed field of characteristic 0 , if $x$ is a point of $\mathcal{X}$ with stabilizer $G_{x}$, then the degree of the 0 -cycle $[x] \in A_{0}(\mathcal{X})$ is $1 /\left|G_{x}\right|$. As a consequence one can compute the integral of an algebraic cohomology class on a complete Deligne-Mumford stack. This theory of integration plays a crucial role in Gromov-Witten, Donaldson-Thomas and other invariants in modern algebraic geometry.

A natural problem is to extend the notion of degree to stacks with nonfinite diagonal. While such stacks are not separated (and hence cannot be proper over the ground field), there is a class of Artin stacks which in many ways behave like complete Deligne-Mumford stacks. Specifically we consider Artin stacks which are generically Deligne-Mumford and have a complete good moduli space (in the sense of [Al]). If $\mathcal{X}$ is such a stack, then there is a well-defined pushforward of Grothendieck groups $p_{*}: K(\mathcal{X}) \rightarrow K(p t)=\mathbb{Z}$, thereby defining Euler characteristics of sheaves on such stacks. Thus it is natural to expect there to be a corresponding pushforward for algebraic cycles as well as a Riemann-Roch theorem relating the $K$-theoretic and the Chow-theoretic pushforward via some analogue of the Chern character.

The purpose of the present paper is to take a first step in this direction by defining the degree of a 0-cycle on a toric Artin stack in the sense of [BCS]. Our definition is based on our previous paper [EM], where we showed that if $\mathcal{X}(\boldsymbol{\Sigma})$ is a toric Artin stack with complete good moduli space $X(\Sigma)$, then there is a canonical

Received by the editors January 10, 2012.

2010 Mathematics Subject Classification. Primary 14M25, 14C15, 14D23.

The first author was partially supported by NSA grant H98230-08-1-0059 while preparing this article. 
birational morphism of toric stacks $\mathcal{X}\left(\boldsymbol{\Sigma}^{\prime}\right) \stackrel{f}{\rightarrow} \mathcal{X}(\boldsymbol{\Sigma})$ such that $\mathcal{X}\left(\boldsymbol{\Sigma}^{\prime}\right)$ is DeligneMumford and such that the induced morphism of the underlying toric varieties is proper and birational. The degree of a 0 -cycle on $\mathcal{X}(\boldsymbol{\Sigma})$ is then defined as the degree of its pullback to the Deligne-Mumford stack $\mathcal{X}\left(\Sigma^{\prime}\right)$.

As an application we define the stacky Euler characteristic of an Artin toric stack to be the degree of the Euler class of the tangent bundle stack, and we give a combinatorial formula for this Euler characteristic in dimension 3.

Along the way we prove that the integral Chow ring of an Artin toric stack equals the Stanley-Reisner ring of the associated fan. Iwanari Iwa] proves this for Deligne-Mumford toric stacks; our proof is different.

\section{TORIC STACKS AND Stanley-REISNeR RINGS}

In this section, we recall the definition of toric stacks and in the process establish the notation we will use for toric stacks. As in Iwa, for simplicity we will restrict our attention to stacky fans for which $N$ is torsion free. A stacky fan $\boldsymbol{\Sigma}=\left(N, \Sigma,\left\{v_{1}, \ldots, v_{n}\right\}\right)$ consists of:

1. a finitely generated free abelian group $N=\mathbb{Z}^{d}$,

2. a (not neccesarily simplicial) fan $\Sigma \subset N_{\mathbb{Q}}$, whose rays we will denote by $\rho_{1}, \ldots, \rho_{n}$,

3. for every $1 \leq i \leq n$, an element $v_{i} \in N$ such that $v_{i}$ lies on the ray $\rho_{i}$.

2.1. Toric stacks via the Cox construction. We now recall the construction [BCS] of the toric stack $\mathcal{X}(\boldsymbol{\Sigma})$ associated to a stacky fan $\boldsymbol{\Sigma}=\left(N, \Sigma,\left\{v_{1}, \ldots, v_{n}\right\}\right)$. Let $S=\mathbb{C}\left[X_{\rho} \mid \rho \in \Sigma(1)\right]$, where $\Sigma(1)$ denotes the rays in $\Sigma$. Define the ideal (of S) $B(\Sigma)=\left(X_{\widehat{\sigma}} \mid \sigma \in \Sigma\right) S$, where $X_{\widehat{\sigma}}=\prod_{\rho \in \Sigma(1) \backslash \sigma} X_{\rho}$. The Cox space $C(\Sigma)$ is defined to be Spec $S \backslash V(B(\Sigma))$, where $V(B(\Sigma))$ is the vanishing locus of the ideal $B(\Sigma)$. Let

$$
G(\boldsymbol{\Sigma})=\left\{\left(t_{\rho}\right)_{\rho \in \Sigma(1)} \in\left(\mathbb{C}^{*}\right)^{n} \mid \prod_{\rho \in \Sigma(1)} t_{\rho}^{\left\langle m, v_{\rho}\right\rangle}=1 \quad \forall m \in M\right\}
$$

where $M=\operatorname{Hom}(N, \mathbb{Z})$ and $\langle\cdot, \cdot\rangle: M \times N \rightarrow \mathbb{Z}$ is the natural pairing. The group $G(\boldsymbol{\Sigma})$ acts on $C(\Sigma)$ by restricting the natural action of $\left(\mathbb{C}^{*}\right)^{n}$ on $C(\Sigma) \subseteq \mathbb{C}^{n}=$ Spec $S$. The toric stack $\mathcal{X}(\boldsymbol{\Sigma})$ associated to the stacky fan $\boldsymbol{\Sigma}=\left(N, \Sigma,\left\{v_{1}, \ldots, v_{n}\right\}\right)$ is the quotient stack $[C(\Sigma) / G(\boldsymbol{\Sigma})]$.

2.2. Chow rings of Artin toric stacks. Our first result is a description of the Chow ring of an Artin toric stack in terms of the combinatorics of its stacky fan.

Definition 2.1 (Iwa, Definition 2.1]). Given a stacky fan $\left.\boldsymbol{\Sigma}=\left(N, \Sigma,\left\{v_{\rho}\right\}_{\rho \in \Sigma(1)}\right\}\right)$, let $S(\Sigma)=\mathbb{Z}\left[x_{\rho} \mid \rho \in \Sigma(1)\right]$. Let $I_{\boldsymbol{\Sigma}}$ be the ideal of $S(\Sigma)$ generated by $\sum_{\rho \in \Sigma(1)}\left\langle m, v_{\rho}\right\rangle x_{\rho}$ as $m$ ranges over $M$. Let $J_{\Sigma}$ be the ideal of $S(\Sigma)$ generated by monomials $x_{\rho_{1}} \cdots x_{\rho_{s}}$ such that the rays $\rho_{1}, \ldots, \rho_{s}$ are not contained in any cone in $\Sigma$ :

$$
J_{\Sigma}=\left\langle x_{\rho_{1}} \cdots x_{\rho_{s}} \mid\left\{\rho_{1}, \ldots, \rho_{s}\right\} \nsubseteq \tau \in \Sigma\right\rangle .
$$

We will call $J_{\Sigma}$ the Stanley-Reisner ideal of $\Sigma$. Following [Iwa, Definition 2.1] (but not exactly the possibly more standard terminology in [CLS, Definition 12.4.10]; see also $\mathrm{MS}$ ), define the Stanley-Reisner ring $S R(\boldsymbol{\Sigma})$ to be

$$
S R(\boldsymbol{\Sigma})=S(\Sigma) /\left(I_{\boldsymbol{\Sigma}}+J_{\Sigma}\right) .
$$


Suppose a group $G$ acts on a smooth scheme $X$, and let $\mathcal{X}=[X / G]$ be the resulting quotient stack. The integral Chow ring $A^{*}(\mathcal{X})$ of $\mathcal{X}$ is the $G$-equivariant Chow ring $A_{G}^{*}(X)$ of $X$ EG98]. We prove that the integral Chow ring of an Artin toric stack $\mathcal{X}(\boldsymbol{\Sigma})$ is the Stanley-Reisner ring of the stacky fan $\boldsymbol{\Sigma}$. Iwanari Iwa. proved the same result for toric Deligne-Mumford stacks. Our proof is different: rather than computing the equivariant Chow groups directly from the definition, we use the excision sequence for equivariant Chow groups.

Proposition 2.2. Let $\boldsymbol{\Sigma}=\left(N, \Sigma,\left\{v_{\rho}\right\}_{\rho \in \Sigma(1)}\right)$ be a stacky fan. There is an isomorphism between the integral Chow ring $A^{*}(\mathcal{X}(\boldsymbol{\Sigma}))$ of the smooth toric Artin stack $\mathcal{X}(\boldsymbol{\Sigma})$ and the Stanley-Reisner ring $S R(\boldsymbol{\Sigma})$, sending $\left[V\left(X_{\rho}\right)\right]_{G(\boldsymbol{\Sigma})} \rightarrow x_{\rho}$. Here $\left[V\left(X_{\rho}\right)\right]_{G(\boldsymbol{\Sigma})} \in A_{G(\boldsymbol{\Sigma})}^{*}(C(\Sigma))=A^{*}(\mathcal{X}(\boldsymbol{\Sigma}))$ denotes the equivariant fundamental class of the divisor $V\left(X_{\rho}\right)$ of $C(\Sigma)$.

Proof. As before, let $S(\Sigma)=\mathbb{Z}\left[x_{\rho} \mid \rho \in \Sigma(1)\right], S=\mathbb{C}\left[X_{\rho} \mid \rho \in \Sigma(1)\right]$, and $G=$ $G(\boldsymbol{\Sigma})$.

The excision sequence in equivariant Chow groups is the exact sequence

$$
A_{G}^{*}(V(B(\Sigma))) \rightarrow A_{G}^{*}(\operatorname{Spec} S) \rightarrow A_{G}^{*}(C(\Sigma)) \rightarrow 0 .
$$

Since Spec $S$ is an affine bundle over pt $=\operatorname{Spec} \mathbb{C}$, we have $A_{G}^{*}(\operatorname{Spec} S)=$ $A_{G}^{*}(\mathrm{pt})=S(\Sigma) / I_{\Sigma}$. By the following lemma, the image of $A_{G}^{*}(V(B(\Sigma)))$ in $A_{G}^{*}(\operatorname{Spec} S)$ is the Stanley-Reisner ideal $J_{\Sigma}$ (equation (1)). Hence $A_{G}^{*}(C(\Sigma))=$ $S(\Sigma) /\left(I_{\boldsymbol{\Sigma}}+J_{\Sigma}\right)=S R(\boldsymbol{\Sigma})$, the Stanley-Reisner ring of $\Sigma$.

Lemma 2.3. Let $B(\Sigma)=\left(X_{\widehat{\sigma}} \mid \sigma \in \Sigma\right) S$ be as in Section 2.1. Then we have a primary decomposition

$$
B(\Sigma)=\bigcap_{\left\{\rho_{1}, \ldots, \rho_{s}\right\} \nsubseteq \tau \in \Sigma}\left(X_{\rho_{1}}, \ldots, X_{\rho_{s}}\right)
$$

where the intersection ranges over sets of rays $\left\{\rho_{1}, \ldots, \rho_{s}\right\}$ that are not contained in some cone in $\Sigma$.

Proof. First we show the " $\subseteq$ " inclusion. Fix $\sigma \in \Sigma$. For any $\left\{\rho_{1}, \ldots, \rho_{s}\right\}$ not contained in a cone in $\Sigma$, there is some $\rho_{i}$ (where $1 \leq i \leq s$ ) such that $\rho_{i} \notin \sigma$ (for otherwise $\left.\left\{\rho_{1}, \ldots, \rho_{s}\right\} \subseteq \sigma\right)$. Hence $X_{\widehat{\sigma}} \in\left(X_{\rho_{1}}, \ldots, X_{\rho_{s}}\right)$, and hence we have proved the " $\subseteq$ " inclusion.

To show the opposite inclusion, let $R I$ denote the ideal on the right-hand side of equation (3). Since $R I$ is a monomial ideal, it suffices to show that monomials in $R I$ belong to $B(\Sigma)$; i.e., we need to show if a monomial $X_{\rho_{1}} \cdots X_{\rho_{s}}$ lies in $R I$, then it lies in $B(\Sigma)$. We will show the contrapositive, namely that if $X_{\rho_{1}} \cdots X_{\rho_{s}} \notin B(\Sigma)$, then $X_{\rho_{1}} \cdots X_{\rho_{s}} \notin R I$. If $X_{\rho_{1}} \cdots X_{\rho_{s}} \notin B(\Sigma)$, then the set $\Sigma(1) \backslash\left\{\rho_{1}, \ldots, \rho_{s}\right\}$ is not contained in any cone of $\Sigma$ (because if $\Sigma(1) \backslash\left\{\rho_{1}, \ldots, \rho_{s}\right\} \subseteq \tau(1)$ for some cone $\tau \in \Sigma$, then taking complements gives $\left\{\rho_{1}, \ldots, \rho_{s}\right\} \supseteq \Sigma(1) \backslash \tau(1)$, and hence $X_{\rho_{1}} \cdots X_{\rho_{s}}$ would be a multiple (in $S$ ) of $X_{\widehat{\tau}} \in B(\Sigma)$ ). If we let $\left\{q_{1}, \ldots, q_{t}\right\}=\Sigma(1) \backslash$ $\left\{\rho_{1}, \ldots, \rho_{s}\right\}$, then $X_{\rho_{1}} \cdots X_{\rho_{s}} \notin\left(X_{q_{1}}, \ldots, X_{q_{t}}\right)$, and hence $X_{\rho_{1}} \cdots X_{\rho_{s}} \notin R I$.

In the remainder of this article, by virtue of the isomorphism $S R(\boldsymbol{\Sigma}) \simeq A^{*}(\mathcal{X}(\boldsymbol{\Sigma}))$ given in Proposition 2.2, we will write $x_{\rho}$ to mean $\left[V\left(X_{\rho}\right)\right]_{G(\boldsymbol{\Sigma})} \in A^{*}(\mathcal{X}(\boldsymbol{\Sigma}))$ (where $\rho \in \Sigma(1))$. 


\section{Integration on COMPlete toric Deligne-Mumford stacks}

In this section, we study intersection theory on complete toric Deligne-Mumford stacks. In this case, we have an isomorphism $\phi: A^{*}(\mathcal{X}(\boldsymbol{\Sigma}))_{\mathbb{Q}} \rightarrow A^{*}(X(\Sigma))_{\mathbb{Q}}[$ EG98], and for the rest of this paper, we will work with the rational (as opposed to integral) Chow ring $A^{*}(\mathcal{X}(\boldsymbol{\Sigma}))_{\mathbb{Q}}$ of toric stacks.

Definition 3.1 $\left(D_{\sigma, \boldsymbol{\Sigma}}\right.$, stacky multiplicity of a cone). Let $N$ be a lattice, let $\sigma$ be the simplicial cone $N_{\mathbb{R}}$, and let $s=\operatorname{dim} \sigma$. Suppose $v_{1}, \ldots, v_{s}$ are elements of $N$ such that $\sigma=\mathbb{R}_{\geq 0}\left\langle v_{1}, \ldots, v_{s}\right\rangle$ (in particular, each $v_{i}$ is a lattice point on exactly one ray of $\sigma)$. Let $N_{\sigma}=\mathbb{Z}\langle N \cap \sigma\rangle$ be the lattice generated by $N \cap \sigma$. We define the (stacky) multiplicity of the stacky cone $\bar{\sigma}=\left(N, \sigma,\left\{v_{1}, \ldots, v_{s}\right\}\right)$ to be the order of $N_{\sigma} / \mathbb{Z}\left\langle v_{1}, \ldots, v_{s}\right\rangle$, and denote this positive integer by $D_{\bar{\sigma}}$. Let $\boldsymbol{\Sigma}=\left(N, \Sigma,\left\{v_{1}, v_{2}, \ldots, v_{n}\right\}\right)$ be a stacky fan and $\sigma$ a simplicial cone in $\Sigma$. Label the $v_{i}$ so that $v_{1}, \ldots, v_{s}$ are on the rays of $\sigma$. Let $\bar{\sigma}=\left(N, \sigma,\left\{v_{1}, \ldots, v_{s}\right\}\right)$. Define the stacky multiplicity of $\sigma$ with respect to $\boldsymbol{\Sigma}$ to be $D_{\bar{\sigma}}$, and denote this quantity by $D_{\sigma, \Sigma}$.

Proposition 3.2 (EG03, p. 3749]). Let $\boldsymbol{\Sigma}$ be a complete simplicial stacky fan, and let $\pi: \mathcal{X}(\boldsymbol{\Sigma}) \rightarrow X(\Sigma)$ be the coarse moduli space of $\mathcal{X}(\boldsymbol{\Sigma})$. Given distinct rays $\rho_{1}, \ldots, \rho_{s}$ of $\Sigma$ forming a cone $\sigma=\left\langle\rho_{1}, \ldots, \rho_{s}\right\rangle$ in $\Sigma$, let $V(\sigma) \subseteq X(\Sigma)$ be the orbit closure associated to $\sigma$. Let $\phi: A^{*}(\mathcal{X}(\Sigma))_{\mathbb{Q}} \rightarrow A^{*}(X(\Sigma))_{\mathbb{Q}}$ be the isomorphism of Chow rings ([EG98, Theorems 3, 4]). Then

$$
\phi\left(x_{\rho_{1}} \cdots x_{\rho_{s}}\right)=\frac{1}{D_{\sigma, \boldsymbol{\Sigma}}}[V(\sigma)],
$$

where $D_{\sigma, \boldsymbol{\Sigma}}$ is the stacky multiplicity of $\sigma$ in $\boldsymbol{\Sigma}$ (Definition 3.1).

3.1. Self-intersections on toric Deligne-Mumford stacks. If $\rho_{1}, \ldots, \rho_{s}$ are not distinct, then to compute $\phi\left(x_{\rho_{1}} \cdots x_{\rho_{s}}\right)$, we can express $x_{\rho_{1}} \cdots x_{\rho_{s}}$ as a rational linear combination of monomials with no factor $x_{\rho}$ appearing more than once, and then we can apply Proposition 3.2 to compute $\phi$. The following theorem gives a procedure for finding such a linear combination.

Theorem 3.3 (Recursive formula for self-intersections). Let $\boldsymbol{\Sigma}=\left(\mathbb{Z}^{d}, \Sigma,\left\{v_{\rho}\right\}_{\rho \in \Sigma(1)}\right)$ be a complete simplicial stacky fan. Let $\rho_{1}, \ldots, \rho_{s}$ be distinct rays in $\Sigma$, and let $a_{1}, \ldots, a_{s}$ be positive integers and assume $a_{i_{0}} \geq 2$ for some $1 \leq i_{0} \leq s$. If $\rho_{1}, \ldots, \rho_{s}$ are not contained in a cone in $\Sigma$, then $\prod_{i=1}^{s} x_{\rho_{i}}=0\left(\right.$ in $A^{*}(\mathcal{X}(\boldsymbol{\Sigma}))$ and hence $\prod_{i=1}^{s} x_{\rho_{i}}^{a_{i}}=0$. Otherwise, fix a (maximal) d-dimensional cone $\sigma=$ $\left\langle\rho_{1}, \ldots, \rho_{s}, \rho_{s+1}, \ldots, \rho_{d}\right\rangle \in \Sigma(d)$ containing $\rho_{1}, \ldots, \rho_{s}$. Let $C=\{\rho \in \Sigma(1) \backslash$ $\left.\left\{\rho_{1}, \ldots, \rho_{d}\right\} \mid\left\langle\rho, \rho_{1}, \ldots, \rho_{s}\right\rangle \in \Sigma\right\}$ (note $C \neq \emptyset$ since $\Sigma$ is complete).

For a ray $\rho \in C$, let $b_{\rho_{i}, \rho}$ be rational numbers such that

$$
v_{\rho}+\sum_{i=1}^{d} b_{\rho_{i}, \rho} v_{\rho_{i}}=0 .
$$

(Such a relation always exists and is unique since $v_{\rho_{1}}, \ldots, v_{\rho_{d}}$ form a basis of $\mathbb{R}^{d}$.) Then

$$
\prod_{i=1}^{s} x_{\rho_{i}}^{a_{i}}=\sum_{\rho \in C} b_{\rho_{i_{0}}, \rho} x_{\rho} x_{\rho_{i_{0}}}^{a_{i_{0}}-1} \prod_{i=1, i \neq i_{0}}^{s} x_{\rho_{i}}^{a_{i}}
$$

in $A^{*}(\mathcal{X}(\boldsymbol{\Sigma}))_{\mathbb{Q}}$. 
Remark 3.4. Note that on the right-hand side of equation (6), the exponent of $x_{\rho_{i_{0}}}$ is one less than on the left-hand side, but for $i \neq i_{0}$ (and $1 \leq i \leq s$ ) the exponent of $x_{\rho_{i}}$ is the same on both sides. Since there was nothing special about $x_{\rho_{1}}$ (other than $a_{1} \geq 2$ ), we can repeatedly apply Theorem 3.3 to express any monomial $\prod_{i=1}^{s} x_{\rho_{i}}^{a_{i}}$ as a rational linear combination of monomials with no factor $x_{\rho}$ appearing more than once. We can then apply Proposition 3.2 to compute $\phi$. Hence Theorem 3.3 gives a method to compute the integral of (or more generally, $\phi$ applied to) any Chow cohomology class on $\mathcal{X}(\boldsymbol{\Sigma})$. We give an example after the proof.

Proof. For notational convenience, assume $i_{0}=1$. Let $P=x_{\rho_{1}}^{a_{1}-1} \prod_{i=2}^{s} x_{\rho_{i}}^{a_{i}}$. For every $m \in M$, we have $\sum_{\rho \in \Sigma(1)}\left\langle m, v_{\rho}\right\rangle x_{\rho}=0$ in the Chow ring $A^{*}(\mathcal{X}(\boldsymbol{\Sigma}))_{\mathbb{Q}}$. Multiplying by $P$ we get

$$
\sum_{\rho \in C}\left\langle m, v_{\rho}\right\rangle x_{\rho} P+\sum_{i=1}^{d}\left\langle m, v_{\rho_{i}}\right\rangle x_{\rho_{i}} P=0,
$$

where we used $x_{\rho} P=0$ for $\rho \notin C \cup\left\{\rho_{1}, \ldots, \rho_{d}\right\}$ (by Proposition 2.2). By equation (5)

$$
v_{\rho}=-\sum_{i=1}^{d} b_{\rho_{i}, \rho} v_{\rho_{i}} .
$$

Substituting this expression for $v_{\rho}$ in the first term of equation (7) gives

$$
\sum_{\rho \in C}\left\langle m,-\sum_{i=1}^{d} b_{\rho_{i}, \rho} v_{\rho_{i}}\right\rangle x_{\rho} P++\sum_{i=1}^{d}\left\langle m, v_{\rho_{i}}\right\rangle x_{\rho_{i}} P=0,
$$

and collecting the coefficients of $\left\langle m, v_{\rho_{i}}\right\rangle$ gives

$$
\sum_{i=1}^{d}\left\langle m, v_{\rho_{i}}\right\rangle\left(x_{\rho_{i}} P-\sum_{\rho \in C} b_{\rho_{i}, \rho} x_{\rho} P\right)=0 .
$$

Equation (9) holds for all $m \in M$. Since $v_{\rho_{1}}, \ldots, v_{\rho_{d}}$ are linearly independent, we conclude that $x_{\rho_{i}} P-\sum_{\rho \in C} b_{\rho_{i}, \rho} x_{\rho} P=0 \in A^{*}(\mathcal{X}(\boldsymbol{\Sigma}))_{\mathbb{Q}}$ for $1 \leq i \leq d$.

In particular, taking $i=1$, we conclude $x_{\rho_{1}} P=\sum_{\rho \in C} b_{\rho_{1}, \rho} x_{\rho} P$, i.e.

$$
\prod_{i=1}^{s} x_{\rho_{i}}^{a_{i}}=\sum_{\rho \in C} b_{\rho_{1}, \rho} x_{\rho} x_{\rho_{1}}^{a_{1}-1} \prod_{i=2}^{s} x_{\rho_{i}}^{a_{i}} .
$$

Example 3.5. Let $v_{1}=(1,0,1), v_{2}=(0,2,1), v_{3}=(-1,0,1), v_{4}=(0,-1,1)$, $v_{5}=(0,0,-1)$. Let $\Sigma \subseteq \mathbb{R}^{3}$ be the fan whose maximal cones are (the $\mathbb{R}_{\geq 0}$-spans of) $\sigma=\left\langle v_{1}, v_{2}, v_{3}, v_{4}\right\rangle$, and for $(i, j) \in\{(1,2),(2,3),(3,4),(1,4)\}, \sigma_{i j 5}=\left\langle v_{i}, v_{j}, v_{5}\right\rangle$. The only non-simplicial cone is $\sigma$, and its star subdivision is formed by splitting it into four 3-cones, each having the ray through $v_{0}=v_{1}+v_{2}+v_{3}+v_{4}=(0,1,4)$. The cones in $\Sigma_{\sigma}(3) \backslash \Sigma$ are $\sigma_{0 i j}=\left\langle v_{0}, v_{i}, v_{j}\right\rangle$ for $(i, j) \in\{(1,2),(2,3),(3,4),(1,4)\}$. Let $\boldsymbol{\Sigma}=\left(\mathbb{Z}^{3}, \Sigma,\left\{v_{1}, v_{2}, v_{3}, v_{4}, v_{5}\right\}\right)$ and $\boldsymbol{\Sigma}_{\sigma}=\left(\mathbb{Z}^{3}, \Sigma_{\sigma},\left\{v_{0}, v_{1}, v_{2}, v_{3}, v_{4}, v_{5}\right\}\right)$ be the corresponding toric stack. For brevity, let $D i j k=D_{\sigma_{i j k}, \boldsymbol{\Sigma}_{\sigma}}$. Computing the determinants of the appropriate triples of lattice vectors $v_{i}$, we get that $D 125=D 235=2$, $D 345=D 145=1, D 012=D 023=7, D 034=D 014=5$.

For brevity let $x_{i}=x_{\left\langle v_{i}\right\rangle} \in A^{*}\left(\mathcal{X}\left(\boldsymbol{\Sigma}_{\mathbf{s}}\right)\right)_{\mathbb{Q}}$. We use Theorem 3.3 to compute $\phi\left(x_{0}^{2}\right)$ and $\phi\left(x_{0}^{3}\right)$. We will extend $v_{0}$ to the basis $v_{0}, v_{1}, v_{2}$. Then $C=\left\{\left\langle v_{3}\right\rangle,\left\langle v_{4}\right\rangle\right\}$. 
A calculation shows that equation (5) reads $v_{3}+\frac{-4}{7} v_{0}+v_{1}+\frac{2}{7} v_{2}=0$ and $v_{4}+$ $\frac{-3}{7} v_{0}+0 \cdot v_{1}+\frac{5}{7} v_{2}=0$. Hence equation (6) reads $x_{0}^{3}=\frac{-4}{7} x_{0}^{2} x_{3}+\frac{-3}{7} x_{0}^{2} x_{4}$. We have to do another iteration to simplify $x_{0}^{2} x_{3}$ and $x_{0}^{2} x_{4}$. This time, we extend the basis $v_{0}, v_{3}$ to $v_{0}, v_{3}, v_{4}$. Then equation (5) becomes $v_{1}+\frac{-2}{5} v_{0}+v_{3}+\frac{-2}{5} v_{4}=0$, and $v_{2}+\frac{-3}{5} v_{0}+0 \cdot v_{3}+\frac{7}{5} v_{4}=0$. Then equation (6) gives $x_{0}^{2} x_{3}=\frac{-3}{5} x_{0} x_{2} x_{3}$ and $x_{0}^{2} x_{4}=\frac{-2}{5} x_{0} x_{1} x_{4}$. Hence $x_{0}^{3}=\frac{12}{35} x_{0} x_{2} x_{3}+\frac{6}{35} x_{0} x_{2} x_{4}$, and we have eliminated all the self-intersections. Then by Proposition 3.2. $\int_{\mathcal{X}\left(\boldsymbol{\Sigma}_{\sigma}\right)} x_{0}^{3}=\phi\left(x_{0}^{3}\right)=\frac{12}{35} \frac{1}{7}+\frac{6}{35} \frac{1}{5}=\frac{102}{1225}$.

Similarly, $x_{0}^{2}=\frac{-4}{7} x_{0} x_{3}+\frac{-3}{7} x_{0} x_{4}$. Applying Proposition 3.2 gives the relation $\left[V\left(\rho_{0}\right)\right]^{2}=\frac{-4}{7}\left[V\left(\left\langle v_{0}, v_{3}\right\rangle\right)\right]+\frac{-3}{7} \frac{1}{5}\left[V\left(\left\langle v_{0}, v_{4}\right\rangle\right)\right]$ in $A^{*}\left(X\left(\Sigma_{\sigma}\right)\right)_{\mathbb{Q}}$ in the rational Chow group of the toric variety $X\left(\Sigma_{\sigma}\right)$.

As a special case of Theorem 3.3. we recover a formula for $x_{\rho_{1}}^{2} x_{\rho_{2}} \cdots x_{\rho_{d-1}}$, equivalent to the one given in [CLS, Prop. 6.4.4]:

Corollary 3.6. Let $\boldsymbol{\Sigma}=\left(\mathbb{Z}^{d}, \Sigma,\left\{v_{\rho}\right\}_{\rho \in \Sigma(1)}\right)$ be a complete simplicial stacky fan. Let $\tau^{+}$and $\tau^{-}$be distinct (maximal) d-dimensional cones of $\Sigma$ such that $\sigma=\tau^{+} \cap \tau^{-}$ is a cone of dimension $d-1$. Let $\rho^{+}$and $\rho^{-}$be the two rays of $\Sigma$ such that $\tau^{+}=\left\langle\rho^{+}, \sigma\right\rangle$ and $\tau^{-}=\left\langle\rho^{-}, \sigma\right\rangle$. Let $\beta^{+}, \beta^{-}, b_{\rho}$ be rational numbers such that we have a relation

$$
\beta^{+} v_{\rho^{+}}+\beta^{-} v_{\rho^{-}}+\sum_{\rho \in \sigma(1)} b_{\rho} v_{\rho}=0 .
$$

Then for any $\rho \in \sigma(1)$,

$$
x_{\rho} x_{\sigma}=\frac{b_{\rho}}{\beta^{+}} x_{\rho^{+}} x_{\sigma}
$$

in $A^{*}(\mathcal{X}(\boldsymbol{\Sigma}))$.

Proof. Simply take $s=d-1, a_{1}=2, a_{i}=1$ for $2 \leq i \leq s$ in Theorem 3.3 ,

Remark 3.7. Applying the ring isomorphism $\phi: A^{*}(\mathcal{X}(\Sigma))_{\mathbb{Q}} \rightarrow A^{*}(X(\Sigma))_{\mathbb{Q}}$ to the result in Corollary 3.6 gives $\phi\left(x_{\rho}\right) \phi\left(x_{\sigma}\right)=\frac{b_{\rho}}{\beta^{+}} \phi\left(x_{\rho^{+}} x_{\sigma}\right)$. By Proposition 3.2, this equality becomes

$$
[V(\rho)] \cdot\left[\frac{1}{D_{\sigma, \boldsymbol{\Sigma}}} V(\sigma)\right]=\frac{b_{\rho}}{\beta^{+}}\left[\frac{1}{D_{\tau^{+}, \boldsymbol{\Sigma}}} V\left(\tau^{+}\right)\right],
$$

which rearranges to the formula given in [CLS, Prop. 6.4.4]:

$$
[V(\rho)] \cdot[V(\sigma)]=\frac{b_{\rho}}{\beta^{+}} \frac{D_{\sigma, \boldsymbol{\Sigma}}}{D_{\tau^{+}, \boldsymbol{\Sigma}}}\left[V\left(\tau^{+}\right)\right],
$$

for any $\rho \in \sigma(1)$.

\subsection{Euler characteristic of toric Deligne-Mumford stacks.}

Definition 3.8. If $\mathcal{X}$ is an $n$-dimensional smooth complete Deligne-Mumford stack, we define the Euler characteristic of $\mathcal{X}$ as $\chi(\mathcal{X}):=\int_{\mathcal{X}} c_{n}(\mathbb{T})$, where $\mathbb{T}$ is the tangent bundle of $\mathcal{X}$.

Remark 3.9. The Euler characteristic we define is a rational number and should not be confused with the orbifold Euler characteristic defined by physicists. There is an orbifold version of the Gauss-Bonet theorem which implies that our definition is equivalent to the following topological definition for stacks of the form $[M / G]$, 
where $G$ is a finite group and $M$ is a manifold. The manifold $M$ has a CW decomposition where the stabilizer has constant order $g_{c}$ on the interior of each cell $c$. Then $\chi([M / G]):=\sum_{c} \frac{(-1)^{\operatorname{dim} c}}{g_{c}}$. Since every Deligne-Mumford stack (with finite stabilizer) is locally of the form $[M / G]$, the definition of a Euler characteristic can be extended to arbitrary Deligne-Mumford stacks, but we do not need this here.

The following is well-known, but we include a proof for lack of a suitable reference.

Proposition 3.10. Let $\boldsymbol{\Sigma}=\left(N, \Sigma,\left\{v_{1}, v_{2}, \ldots, v_{n}\right\}\right)$ be a complete simplicial stacky fan (i.e. $\Sigma$ is simplicial and complete). Let $\Sigma_{\max }$ denote the set of maximal cones in $\Sigma$. For a maximal cone $\sigma \in \Sigma_{\max }$, let $D_{\sigma}$ denote the multiplicity of $\sigma$. Then

$$
\chi(\mathcal{X}(\boldsymbol{\Sigma}))=\sum_{\sigma \in \Sigma_{\max }} \frac{1}{D_{\sigma, \boldsymbol{\Sigma}}} .
$$

Proof. Let $\mathcal{X}=\mathcal{X}(\boldsymbol{\Sigma})=[C / G]$, where $C=C(\Sigma) \subseteq \mathbb{A}^{n}=\operatorname{Spec} \mathbb{C}\left[X_{1}, \ldots, X_{n}\right]$ is the Cox space of $\Sigma$ and $G=G(\Sigma)$ is as in Section 2.1. The coarse moduli space of $\mathcal{X}$ is $X=X(\Sigma)$, the toric variety associated to $\Sigma$. The equivariant tangent bundle $T_{C}$ is $\oplus \mathcal{O}\left(X_{i}\right)$ (EG03, p. 3751]), so

$$
\chi(\mathcal{X}(\boldsymbol{\Sigma}))=\int_{C} \prod_{i=1}^{n}\left(1+x_{i}\right)=\int_{X} \phi\left(\prod\left(1+x_{i}\right)\right) .
$$

If $v_{i_{1}}, \ldots, v_{i_{s}}$ are not contained in a cone of $\Sigma$, then $\left[V\left(X_{i_{1}}, \ldots, X_{i_{s}}\right)\right]_{G}=0$ in $A_{G}^{*}(C)$ (Proposition 2.2). Hence if we expand the product $\prod_{i=1}^{n}\left(1+x_{i}\right)$, the only monomials $x_{i_{1}} \cdots x_{i_{s}}$ that can contribute to $\int_{X} \phi\left(\prod 1+x_{i}\right)$ are ones where $v_{i_{1}}, \ldots, v_{i_{s}}$ span a maximal cone (we require maximal cones since the integral only considers the dimension 0 part of the cycle). In this case, the image of $V\left(X_{i_{1}}, \ldots, X_{i_{s}}\right)$ is a point in $X(\Sigma)$, and Proposition 3.2 tells us to assign a factor of $\frac{1}{D_{\sigma, \Sigma}}$ to the class [pt] of this point. The usual identification of $A_{0}(X(\Sigma))$ with $\mathbb{Z}$ sending $[\mathrm{pt}] \in A^{*}(X(\Sigma))$ to $1 \in \mathbb{Z}$ gives the result.

Remark 3.11. Note that Proposition 3.10 recovers the result (e.g. CLS, Theorem 12.3.9]) that the Euler characteristic of a smooth toric variety is the number of maximal cones.

The following result expresses how the Euler characteristic increases after a stacky star subdivision [EM, Definition 4.1] of a simplicial stacky fan.

Lemma 3.12. Let $\boldsymbol{\Sigma}=\left(\mathbb{Z}^{d}, \Sigma,\left\{v_{1}, \ldots, v_{n}\right\}\right)$ be a complete simplicial stacky fan and let $\sigma$ be a cone in $\Sigma$. Let $\boldsymbol{\Sigma}_{\sigma}=\left(\mathbb{Z}^{d}, \Sigma_{\sigma},\left\{v_{0}, v_{1}, \ldots, v_{n}\right\}\right)$ be the stacky fan formed by stacky star subdivision of $\boldsymbol{\Sigma}$ with respect to $\sigma$. Then $\chi\left(\mathcal{X}\left(\boldsymbol{\Sigma}_{\sigma}\right)\right)=\chi(\mathcal{X}(\boldsymbol{\Sigma}))+\frac{s-1}{D_{\sigma, \boldsymbol{\Sigma}}}$, where $s=\operatorname{dim} \sigma$.

Proof. Label the $v_{i}$ such that $\sigma=\left\langle v_{1}, \ldots, v_{s}\right\rangle$. Hence $v_{0}=v_{1}+\cdots+v_{s}$. For $1 \leq i \leq s$, let $\sigma_{i}=\left\langle v_{1}, \ldots, \widehat{v_{i}}, \ldots, v_{s}\right\rangle$ be the cone of $\Sigma_{\sigma}$ spanned by $v_{0}$ and all the rays of $\sigma$ except for $\left\langle v_{i}\right\rangle$. Then by properties of determinants, we have $D_{\sigma_{i}, \boldsymbol{\Sigma}_{\sigma}}=D_{\sigma, \boldsymbol{\Sigma}}$. Then apply Proposition 3.10 . 


\section{Integration on ARtin toric Stacks}

We now come to the main definitions of the paper. Let $\boldsymbol{\Sigma}$ be a (not necessarily simplicial) stacky fan and let $\mathcal{X}(\boldsymbol{\Sigma})$ be the associated Artin toric stack. By EM, Theorem 5.2] there is a simplicial stacky fan $\boldsymbol{\Sigma}^{\prime}$ canonically obtained from $\boldsymbol{\Sigma}$ by stacky star subdivisions and a commutative diagram of stacks and toric varieties

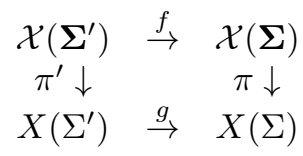

where $f$ is birational, $\pi^{\prime}$ is finite and $g$ is proper and birational.

Definition 4.1. If $\alpha \in A^{*}(\mathcal{X}(\boldsymbol{\Sigma}))$, define $\pi_{*} \alpha:=g_{*} \pi_{*}^{\prime} f^{*} \alpha$. In particular, if the associated toric variety $X(\Sigma)$ is complete, we define for any $\alpha \in A^{d}(\mathcal{X}(\boldsymbol{\Sigma}))$

$$
\int_{\mathcal{X}(\boldsymbol{\Sigma})} \alpha:=\int_{\mathcal{X}\left(\boldsymbol{\Sigma}^{\prime}\right)} f^{*} \alpha
$$

4.1. The Euler characteristic of an Artin toric stack with complete good moduli space. We now turn to the definition of the Euler characteristic of an Artin toric stack. If $\mathcal{X}=[X / G]$ is a smooth Artin stack, then the tangent bundle stack to $\mathcal{X}$ equals the vector bundle stack $\mathbb{T} \mathcal{X}=[T X / \operatorname{Lie}(G)]$. The restriction of $\mathbb{T} \mathcal{X}$ to the open set $\mathcal{X}^{D M}$ where $\mathcal{X}$ is Deligne-Mumford is the usual tangent bundle to $\mathcal{X}^{D M}$. By identifying the Chow groups of $\mathcal{X}$ with the $G$-equivariant Chow groups of $X$, we may define the Chern series of $\mathbb{T} \mathcal{X}$ as the formal series $c_{t}(\mathbb{T} \mathcal{X})=$ $c_{t}(T X) c_{t}(\operatorname{Lie}(G))^{-1} \in \prod_{i=0}^{\infty} A_{G}^{i}(X) \otimes \mathbb{Z}[[t]]$. Moreover, when $G$ is diagonalizable the Lie algebra of $G$ is a trivial $G$-module, so $c_{t}(\operatorname{Lie}(G))=1$ and thus the Chern series of $\mathbb{T} \mathcal{X}$ is actually a polynomial.

We can extend the definition of the Euler characteristic to toric Artin stacks.

Definition 4.2. Let $\boldsymbol{\Sigma}$ be a stacky fan and suppose that the associated toric variety $X(\Sigma)$ has dimension $d$. Then we set $\chi(\mathcal{X}(\boldsymbol{\Sigma})):=\int_{\mathcal{X}(\boldsymbol{\Sigma})} c_{d}(\mathbb{T} \mathcal{X})$.

4.2. Formulas for Euler characteristics of 3-dimensional toric Artin stacks. As an application of Theorem 3.3, we derive a formula for the Euler characteristic of a 3-dimensional toric Artin stack.

Lemma 4.3. Let $\boldsymbol{\Sigma}=\left(\mathbb{Z}^{3}, \Sigma,\left\{v_{1}, \ldots, v_{n}\right\}\right)$ be a 3-dimensional complete stacky fan with only one nonsimplicial cone, call it $\sigma$, and label the $v_{i}$ so that $\sigma=$ $\mathbb{R}_{\geq 0}\left\langle v_{1}, \ldots, v_{s}\right\rangle$. Let $\boldsymbol{\Sigma}_{\sigma}=\left(\mathbb{Z}^{3}, \Sigma_{\sigma},\left\{v_{0}, v_{1}, \ldots, v_{n}\right\}\right)$ be the stacky fan formed by the stacky star subdivision of $\boldsymbol{\Sigma}$ with respect to $\sigma$, and let $f: \mathcal{X}\left(\boldsymbol{\Sigma}_{\sigma}\right) \rightarrow \mathcal{X}(\boldsymbol{\Sigma})$ be the induced morphism. For $0 \leq i \leq n$, let $y_{i} \in A^{*}\left(\mathcal{X}\left(\boldsymbol{\Sigma}_{\sigma}\right)\right)$ be the equivariant fundamental class of the coordinate hyperplanes restricted to the Cox space $C\left(\Sigma_{\sigma}\right)$. Let $\Sigma_{\sigma}(3) \backslash \Sigma(3)$ be the set of maximal cones in $\Sigma_{\sigma}$ that are not in $\Sigma$. Then the difference between the Euler characteristic of $\mathcal{X}(\boldsymbol{\Sigma})$ and the Euler characteristic of its simplicialization $\mathcal{X}\left(\boldsymbol{\Sigma}_{\sigma}\right)$ is given by

$$
\begin{aligned}
& \chi(\mathcal{X}(\boldsymbol{\Sigma}))-\chi\left(\mathcal{X}\left(\boldsymbol{\Sigma}_{\sigma}\right)\right) \\
& =\sum_{\tau \in \Sigma_{\sigma}(3) \backslash \Sigma(3)} \frac{s-3}{D_{\tau, \Sigma_{\sigma}}}+\left(\begin{array}{c}
s-1 \\
2
\end{array}\right) \int_{\mathcal{X}\left(\boldsymbol{\Sigma}_{\sigma}\right)} y_{0}^{2}\left(\sum_{1 \leq i \leq s} y_{i}\right)+\left(\begin{array}{c}
s \\
3
\end{array}\right) \int_{\mathcal{X}\left(\boldsymbol{\Sigma}_{\sigma}\right)} y_{0}^{3} .
\end{aligned}
$$


Proof. With the notation as in Section 2.1, for $1 \leq i \leq s$, we have $f^{*}\left(x_{i}\right)=$ $y_{0}+y_{i}$. Also, for $i \geq s, f^{*}\left(x_{i}\right)=y_{i}$. Then $\chi(\mathcal{X}(\boldsymbol{\Sigma}))=\int_{\mathcal{X}\left(\boldsymbol{\Sigma}_{\sigma}\right)} f^{*}\left(\prod_{i=1}^{n}\left(1+x_{i}\right)\right)=$ $\int_{\mathcal{X}\left(\boldsymbol{\Sigma}_{\sigma}\right)}\left(\prod_{i=1}^{s}\left(1+y_{0}+y_{i}\right)\right) \prod_{i>s}\left(1+y_{i}\right)$.

By Proposition 2.2 and equations (2) and (11), we have that $y_{i_{1}} \cdots y_{i_{t}}=0$ in $A_{G}^{*}(C)$ if $v_{i_{1}}, \ldots, v_{i_{t}}$ are not contained in a cone. In particular, $y_{0} y_{i}=0$ for any $i>s$. Hence when we expand $\left(\prod_{i=1}^{s}\left(1+y_{0}+y_{i}\right)\right) \prod_{i>s}\left(1+y_{i}\right)$ and collect the degree 3 , we get (note that some monomials in the following equation are zero)

$$
\int_{\mathcal{X}\left(\boldsymbol{\Sigma}_{\sigma}\right)}\left(\sum_{0<i<j<k} y_{i} y_{j} y_{k}+\sum_{0<j<k \leq s}\left(\begin{array}{c}
s-2 \\
1
\end{array}\right) y_{0} y_{j} y_{k}+\sum_{0<k \leq s}\left(\begin{array}{c}
s-1 \\
2
\end{array}\right) y_{0}^{2} y_{k}+\left(\begin{array}{l}
s \\
3
\end{array}\right) y_{0}^{3}\right) \text {. }
$$

We have $\left(\sum_{0<i<j<k} y_{i} y_{j} y_{k}\right)+\left(\sum_{0<j<k \leq s} y_{0} y_{j} y_{k}\right)=\chi\left(\mathcal{X}\left(\boldsymbol{\Sigma}_{\sigma}\right)\right)$.

Remark 4.4. The above proof easily extends to the case when $\Sigma$ has more than one nonsimplicial cone. Suppose $\sigma_{1}$ and $\sigma_{2}$ are two different nonsimplicial cones of $\Sigma$. Then the above proof shows that the equivariant fundamental class of the exceptional divisor of the blowup of $V\left(\sigma_{1}\right)$ (resp. $V\left(\sigma_{2}\right)$ ) has nonzero product only with divisors coming from rays of $\sigma_{1}$ (resp. $\left.\sigma_{2}\right)$.

By applying Theorem 3.3, we can give a combinatorial formula to the selfintersection integrals on the right-hand side of equation (11).

Fix $i$ satisfying $1 \leq i \leq s$. We will give a formula for $\int_{\mathcal{X}\left(\boldsymbol{\Sigma}_{\sigma}\right)} y_{0}^{2} y_{i}$. Let $v_{i}^{+}$and $v_{i}^{-}$ be the two lattice vectors (among $v_{1}, \ldots, v_{s}$ ) such that $\tau_{i}^{+}=\mathbb{R}_{\geq 0}\left\langle v_{i}^{+}, v_{0}, v_{i}\right\rangle$ and $\tau_{i}^{-}=\mathbb{R}_{\geq 0}\left\langle v_{i}^{-}, v_{0}, v_{i}\right\rangle$ are the two maximal cones in $\Sigma_{\sigma}$ having $\gamma_{i}=\mathbb{R}_{\geq 0}\left\langle v_{0}, v_{i}\right\rangle$ as a common face. Since $\tau_{i}^{+}$and $\tau_{i}^{-}$are simplicial, there is a relation

$$
\beta_{i}^{+} v_{i}^{+}+b_{0, i} v_{0}+b_{i} v_{i}+\beta_{i}^{-} v_{i}^{-}=0,
$$

where $\beta_{i}^{+}, b_{0, i}, b_{i}, \beta_{i}^{-}$are rational numbers. Then by Corollary 3.6.

$$
\int_{\mathcal{X}\left(\boldsymbol{\Sigma}_{\sigma}\right)} y_{0}^{2} y_{i}=\frac{b_{0, i}}{\beta_{i}^{+} D_{\tau_{i}^{+}, \boldsymbol{\Sigma}_{\sigma}}}=\frac{b_{0, i}}{\beta_{i}^{-} D_{\tau_{i}^{-}, \boldsymbol{\Sigma}_{\sigma}}} .
$$

Finally, to compute the triple self-intersection integral $\int_{\mathcal{X}\left(\boldsymbol{\Sigma}_{\sigma}\right)} y_{0}^{3}$, one could use Theorem 3.3. However, because we have restricted the dimension of $\Sigma$ to 3, we can instead use a relation of rational equivalence and reduce the computation to the case of double self-intersection integrals just computed. Namely, pick some $m_{\sigma} \in M$ such that $\left\langle m_{\sigma}, v_{0}\right\rangle \neq 0$ (there exists such an $m_{\sigma}$ since $v_{0} \neq 0$ since $v_{0}$ lies in the interior of $\sigma)$. Then the rational equivalence relation $\operatorname{div}\left(\chi^{m_{\sigma}}\right)=0$ can be written as $\sum_{0 \leq i \leq n}\left\langle m_{\sigma}, v_{i}\right\rangle y_{i}=0$. Hence

$$
y_{0}=\frac{-1}{\left\langle m_{\sigma}, v_{0}\right\rangle} \sum_{1 \leq i \leq n}\left\langle m_{\sigma}, v_{i}\right\rangle y_{i} .
$$

Multiplying by $y_{0}^{2}$ and using the fact that $y_{0} y_{i}=0$ for $i>s$ gives

$$
\int_{\mathcal{X}\left(\boldsymbol{\Sigma}_{\sigma}\right)} y_{0}^{3}=\frac{-1}{\left\langle m_{\sigma}, v_{0}\right\rangle} \int_{\mathcal{X}\left(\boldsymbol{\Sigma}_{\sigma}\right)} \sum_{1 \leq i \leq s}\left\langle m_{\sigma}, v_{i}\right\rangle y_{0}^{2} y_{i} .
$$


Using Remark 4.4, we combine the above formulas to deduce the following result:

Theorem 4.5. Let $\boldsymbol{\Sigma}=\left(\mathbb{Z}^{3}, \Sigma,\left\{v_{\rho}\right\}_{\rho \in \Sigma(1)}\right)$ be a 3-dimensional complete stacky fan. Let $N S$ denote the set of nonsimplicial cones of $\Sigma$. Let

$$
\boldsymbol{\Sigma}_{\text {simp }}=\left(\mathbb{Z}^{3}, \Sigma_{\text {simp }},\left\{v_{\rho}\right\}_{\rho \in \Sigma_{\text {simp }}(1)}\right)
$$

be the stacky fan formed by the stacky star subdivision of $\boldsymbol{\Sigma}$ with respect to the cones in $N S$, and let $f: \mathcal{X}\left(\boldsymbol{\Sigma}_{\mathbf{s i m p}}\right) \rightarrow \mathcal{X}(\boldsymbol{\Sigma})$ be the induced morphism.

For $\sigma \in N S$, let $s_{\sigma}=|\sigma(1)|$ be the number of rays in $\sigma$, let $v_{\sigma}=\sum_{\rho \in \sigma(1)} v_{\rho}$, let $m_{\sigma} \in M$ be such that $\left\langle m_{\sigma}, v_{\sigma}\right\rangle \neq 0$, and regard $\Sigma_{\sigma} \subseteq \Sigma_{\text {simp. }}$. For each $\rho \in$ $\sigma(1)$ let $v_{\rho}^{+}$and $v_{\rho}^{-}$be the two lattice vectors such that $\tau_{\rho}^{+}=\mathbb{R}_{\geq 0}\left\langle v_{\rho}^{+}, v_{\sigma}, v_{\rho}\right\rangle$ and $\tau_{\rho}^{-}=\mathbb{R}_{\geq 0}\left\langle v_{\rho}^{-}, v_{\sigma}, v_{\rho}\right\rangle$ are the two maximal cones in $\Sigma_{\sigma}$ having $\gamma_{\rho}=\mathbb{R}_{\geq 0}\left\langle v_{\sigma}, v_{\rho}\right\rangle$ as a common face. Let $\beta_{\rho}^{+}, b_{\sigma, \rho}, b_{\rho}, \beta_{\rho}^{-}$be rational numbers such that

$$
\beta_{\rho}^{+} v_{\rho}^{+}+b_{\sigma, \rho} v_{\sigma}+b_{\rho} v_{\rho}+\beta_{\rho}^{-} v_{\rho}^{-}=0 .
$$

Let $\Sigma_{\sigma}(3) \backslash \Sigma(3)$ be the set of maximal cones in $\Sigma_{\sigma}$ that are not in $\Sigma$. Let $D_{\sigma, \boldsymbol{\Sigma}}$ be as in Definition 3.1. Then the difference between the Euler characteristic of $\mathcal{X}(\boldsymbol{\Sigma})$ and the Euler characteristic of its simplicialization $\mathcal{X}\left(\boldsymbol{\Sigma}_{\mathbf{s i m p}}\right)$ is given by

$$
\begin{aligned}
& \chi(\mathcal{X}(\boldsymbol{\Sigma}))-\chi\left(\mathcal{X}\left(\boldsymbol{\Sigma}_{\text {simp }}\right)\right) \\
& =\sum_{\sigma \in N S}\left(\sum_{\tau \in \Sigma_{\sigma}(3) \backslash \Sigma(3)} \frac{s_{\sigma}-3}{D_{\tau, \Sigma_{\sigma}}}+\sum_{\rho \in \sigma(1)}\left(\left(\begin{array}{c}
s_{\sigma}-1 \\
2
\end{array}\right)-\left(\begin{array}{c}
s_{\sigma} \\
3
\end{array}\right) \frac{\left\langle m_{\sigma}, v_{\rho}\right\rangle}{\left\langle m_{\sigma}, v_{\sigma}\right\rangle}\right) \frac{b_{\sigma, \rho}}{\beta_{\rho}^{+} D_{\tau_{\rho}^{+}, \boldsymbol{\Sigma}_{\sigma}}}\right) .
\end{aligned}
$$

As an example, we use this formula to compute the Euler characteristic of the nonsimplicial toric stack considered in Example 3.5.

Example 4.6. We continue with Example 3.5, and let $f: \mathcal{X}\left(\boldsymbol{\Sigma}_{\sigma}\right) \rightarrow \mathcal{X}(\boldsymbol{\Sigma})$ be the morphism induced by the stacky star subdivision of $\Sigma$ with respect to $\sigma$. The multiplicities of the maximal cones are $D 125=D 235=2, D 345=D 145=1$, $D 012=D 023=7, D 034=D 014=5$. Hence by Proposition 3.10 , we have

$$
\chi\left(\mathcal{X}\left(\boldsymbol{\Sigma}_{\sigma}\right)\right)=\frac{1}{7}+\frac{1}{7}+\frac{1}{5}+\frac{1}{5}+\frac{1}{2}+\frac{1}{2}+\frac{1}{1}+\frac{1}{1}=\frac{129}{35} .
$$

Also, $N S=\{\sigma\}$ and $s_{\sigma}=4$, so

$$
\sum_{\tau \in \Sigma_{\sigma}(3) \backslash \Sigma(3)} \frac{s_{\sigma}-3}{D_{\tau, \Sigma_{\sigma}}}=\frac{1}{7}+\frac{1}{7}+\frac{1}{5}+\frac{1}{5}=\frac{24}{35} .
$$

Since $v_{\sigma}=v_{0}=(0,1,4)$, we may take $m_{\sigma}=(0,1,0)$, so $\left\langle m_{\sigma}, v_{\sigma}\right\rangle=1$. There are four rays in $\sigma(1)$, namely $\rho_{i}=\mathbb{R}_{\geq 0}\left\langle v_{i}\right\rangle$ for $i=1,2,3,4$, and for each of them we compute

$$
\left(\left(\begin{array}{c}
s_{\sigma}-1 \\
2
\end{array}\right)-\left(\begin{array}{c}
s_{\sigma} \\
3
\end{array}\right) \frac{\left\langle m_{\sigma}, v_{\rho}\right\rangle}{\left\langle m_{\sigma}, v_{\sigma}\right\rangle}\right) \frac{b_{\sigma, \rho}}{\beta_{\rho}^{+} D_{\tau_{\rho}^{+}, \Sigma_{\sigma}}} .
$$

For $\rho=\rho_{1}$, we have $\gamma_{\rho_{1}}=\mathbb{R}_{\geq 0}\left\langle v_{0}, v_{1}\right\rangle$, take $v_{\rho}^{+}=v_{2}, v_{\rho}^{-}=v_{4}$, and set $\beta_{\rho}^{+}=1$. Then equation (15) becomes $v_{2}+b_{\sigma, \rho_{1}} v_{0}+b_{\rho_{1}} v_{1}+\beta_{4} v_{4}=0$. Substituting in the coordinates of each $v_{i}$, this equation becomes a system of three equations in the three unknowns $b_{\sigma, \rho_{1}}, b_{\rho_{1}}, \beta_{4}$. Solving we get $b_{\sigma, \rho_{1}}=\frac{-3}{5}$ (and $b_{\rho_{1}}=0, \beta_{4}=\frac{7}{5}$, but we won't need them). Hence for $\rho=\rho_{1}$, equation (16) becomes

$$
\left(\left(\begin{array}{c}
4-1 \\
2
\end{array}\right)-\left(\begin{array}{l}
4 \\
3
\end{array}\right) \frac{0}{1}\right) \frac{\frac{-3}{5}}{1 \cdot 7}=\frac{-9}{35} \text {. }
$$


Similarly, computations for $\rho=\rho_{2}, \rho_{3}, \rho_{4}$ yield $\frac{20}{49}, \frac{-9}{35}, \frac{-14}{25}$, respectively.

Hence the Euler characteristic $\chi(\mathcal{X}(\boldsymbol{\Sigma}))=\frac{129}{35}+\frac{24}{35}-\frac{9}{35}+\frac{20}{49}-\frac{9}{35}-\frac{14}{25}=\frac{4539}{1225}$.

\section{REFERENCES}

[Al] Jarod Alper, Good moduli spaces for Artin stacks, math.AG/08042242 (2008).

[BCS] Lev A. Borisov, Linda Chen, and Gregory G. Smith, The orbifold Chow ring of toric Deligne-Mumford stacks, J. Amer. Math. Soc. 18 (2005), no. 1, 193-215 (electronic), DOI 10.1090/S0894-0347-04-00471-0. MR2114820 (2006a:14091)

[CLS] David A. Cox, John B. Little, and Henry K. Schenck, Toric varieties, Graduate Studies in Mathematics, vol. 124, American Mathematical Society, Providence, RI, 2011. MR2810322 (2012g:14094)

[EG98] Dan Edidin and William Graham, Equivariant intersection theory, Invent. Math. 131 (1998), no. 3, 595-634, DOI 10.1007/s002220050214. MR1614555 (99j:14003a)

[EG03] Dan Edidin and William Graham, Riemann-Roch for quotients and Todd classes of simplicial toric varieties, Comm. Algebra 31 (2003), no. 8, 3735-3752, DOI 10.1081/AGB120022440. Special issue in honor of Steven L. Kleiman. MR2007382 (2004h:14015)

[EM] Dan Edidin and Yogesh More, Partial desingularizations of good moduli spaces of Artin toric stacks, Michigan Math. J. 61 (2012), no. 3, 451-474, DOI 10.1307/mmj/1347040252. MR.2975255

[Gil] Henri Gillet, Intersection theory on algebraic stacks and Q-varieties, Proceedings of the Luminy conference on algebraic $K$-theory (Luminy, 1983), 1984, pp. 193-240, DOI 10.1016/0022-4049(84)90036-7. MR772058 (86b:14006)

[Kre] Andrew Kresch, Cycle groups for Artin stacks, Invent. Math. 138 (1999), no. 3, 495-536, DOI 10.1007/s002220050351. MR1719823 (2001a:14003)

[Iwa] Isamu Iwanari, Integral chow rings of toric stacks, Int. Math. Res. Not. IMRN 24 (2009), 4709-4725, DOI 10.1093/imrn/rnp110. MR2564373 (2010m:14003)

[MS] Ezra Miller and Bernd Sturmfels, Combinatorial commutative algebra, Graduate Texts in Mathematics, vol. 227, Springer-Verlag, New York, 2005. MR 2110098 (2006d:13001)

[Vis] Angelo Vistoli, Intersection theory on algebraic stacks and on their moduli spaces, Invent. Math. 97 (1989), no. 3, 613-670, DOI 10.1007/BF01388892. MR1005008 (90k:14004)

Department of Mathematics, University of Missouri-Columbia, Columbia, Missouri 65211

E-mail address: edidind@missouri.edu

Department of Mathematics, Suny College at Old Westbury, Old Westbury, NEW YORK 11568

E-mail address: yogeshmore80@gmail.com 